\title{
Minimizing the Makespan in Flowshop Scheduling for Sustainable Rubber Circular Manufacturing
}

\author{
Peng-Yeng Yin ${ }^{1,2}, *$, Hsin-Min Chen ${ }^{2}$, Yi-Lung Cheng ${ }^{3}$, Ying-Chieh Wei ${ }^{2}$, Ya-Lin Huang ${ }^{1}$ and Rong-Fuh Day ${ }^{1}$ \\ 1 Department of Information Management, National Chi Nan University, No. 1, University Rd., Puli, \\ Nantou 54561, Taiwan; s105213018@mail1.ncnu.edu.tw (Y.-L.H.); rfday@ncnu.edu.tw (R.-F.D.) \\ 2 Institute of Strategy and Development of Emerging Industry, National Chi Nan University, No. 1, \\ University Rd., Puli, Nantou 54561, Taiwan; tiyaling@gmail.com (H.-M.C.); \\ s103245908@ncnu.edu.tw (Y.-C.W.) \\ 3 Department of Electrical Engineering, National Chi Nan University, No. 1, University Rd., Puli, \\ Nantou 54561, Taiwan; yjcheng@ncnu.edu.tw \\ * Correspondence: pyyin@ncnu.edu.tw; Tel.: +886-49-291-0960
}

check for updates

Citation: Yin, P.-Y.; Chen, H.-M.; Cheng, Y.-L.; Wei, Y.-C.; Huang, Y.-L.; Day, R.-F. Minimizing the Makespan in Flowshop Scheduling for Sustainable Rubber Circular Manufacturing. Sustainability 2021, 13, 2576. https://doi.org/10.3390/ su13052576

Academic Editor: Grigorios

L. Kyriakopoulos

Received: 25 January 2021

Accepted: 22 February 2021

Published: 28 February 2021

Publisher's Note: MDPI stays neutral with regard to jurisdictional claims in published maps and institutional affiliations.

Copyright: (c) 2021 by the authors. Licensee MDPI, Basel, Switzerland. This article is an open access article distributed under the terms and conditions of the Creative Commons Attribution (CC BY) license (https:/ / creativecommons.org/licenses/by/ $4.0 /)$.

\begin{abstract}
It is estimated that 1 billion waste tires are generated every year across the globe, yet only $10 \%$ are being processed, and much rubber waste is yielded during manufacturing. These waste tires and rubber scraps are poisonous to the environment when processed via incineration and landfill. Rubber circular manufacturing is an effective solution that reduces not only rubber waste but also raw material costs. In this paper we propose a two-line flowshop model for the circular rubber manufacturing problem (CRMP), where the job sequence of two production lines is appropriately aligned to obtain the shortest makespan while guaranteeing that sufficient rubber waste yielded in the first line is ready to be reused for circular production in the second line. A genetic algorithm (GA) is developed, and the design of its genetic operations is customized to the CRMP context to achieve efficient and effective evolution. The experimental results with both real and synthetic datasets show that the GA significantly surpasses two heuristics in the literature by delivering the minimum makespan, which is 3.4 to $11.2 \%$ shorter than those obtained by the two competing methods.
\end{abstract}

Keywords: flowshop scheduling; circular economy; rubber circular manufacturing; heuristic; genetic algorithm

\section{Introduction}

Circular economy [1] is a new recycling economic ecosystem that reduces wastes or emissions during manufacturing and end-use of the products. It stems from the idea that everything produced or consumed in the manufacturing and end-use stages of the products should be part of the economy. The ecosystem of life-time production shifts from open production to closed-loop production, facilitating zero waste and sustainable development. The traditional open production follows a linear fashion, which goes through various stages of the product life, that is, resource acquisition, production, distribution, consumption, and waste. The waste generated at the end of the process is disposed, if not well processed, and may spoil the environment. The closed-loop production in a circular economy, on the other hand, redesigns the product life such that the end-of-life product, which can be reused or repaired, gets into consumption again. Those products that cannot be reused or repaired are made degradable or compostable and reenter the product life as recycled raw materials. This kind of reuse, repair, and recycle (3R) constitutes the principal elements in a circular economy. It requires deliberated technologies for all stages of product life to ensure the feasibility of 3R designs [2]. A comprehensive review was presented in [3], which proposed a circular economy framework and a practical implementation strategy. The framework classifies existing references on aspects of waste generation, resource scarcity, and sustaining economic benefits. The proposed implementation strategy combines top-down 
and bottom-up approaches. Kumar et al. [4] conducted a questionnaire for manufacturing companies in the UK and EU. With 63 responses from socio-political, economic, legal, and environmental perspectives, the authors identified a number of benefits, opportunities, and barriers to circular economy implementation in UK and EU. A conceptual framework of the circular supply chain was presented in [5] where four dimensions were identified to support the development of the new supply chain to capture additional values. The focus of the review in [6] is particularly on the equipment and machinery sector. Multiple strategies proposed in the literature are clustered to build a homogeneous approach for understanding the standards and strategies. A strategy characterization framework is proposed for the selection of the best strategy for equipment production. It was pointed out in [7] that the inclusion of appropriate information technologies such as Industry 4.0 and Internet of Things (IoT) can facilitate a better practice of circular economy. The deployment of IoT enables the collection of all relevant production data to better predict material demands at every production stage such that ideal zero waste (in terms of materials, storage, and logistics) can be achieved. By implementing Industry 4.0 with assistive artificial intelligence, the production system can diagnose abnormal reactions in real time and troubleshoot problems, so as to reduce the gap between production reality and expectations. As indicated in [8], the definition of circular economy is well accepted in considering the interests and expectations of all stakeholders. Waste minimization is achieved through better process, material, and product designs in the industrial system that is restorative by design.

Circular economy is closely related to green economy, which focuses on reducing environmental damages and promoting sustainable development. The United Nations Environment Programme (UNEP) reported that the green economy must not only be efficient but also low-carbon, resource efficient, and socially inclusive [9]. This implies that production conforming to green economy must ideally produce no waste, and if the waste is inevitable, it should be reused or recycled as part of the resource to make it efficient. To this end, each individual or government of all levels in the society has the right and obligation to consider the natural environment and sustainable ecosystem as economical assets. Many countries and non-government organizations have set forth eco-labelling to recognize green products and services. This green trading ecosystem brings new challenges and opportunities. As a response to this call, the Ministry of Economic Affairs, Taiwan, launched the Green Trade Project Office (GTPO) in 2011 to promote the cross-national exchange of goods, services, resources, and credits that contribute to ecological sustainability. Among others, the energy efficiency, renewable energy, lowcarbon transportation, environmental protection equipment, and sustainable materials are important green products or services [10].

The traditional tire production model generates a huge amount of waste and is unable to recover the end-of-life products. For example, it is estimated that 1 billion waste tires are generated every year across the globe, yet only $10 \%$ of them are being processed [11], and much scrap rubber is cut off during manufacturing. The poisonous materials contained in waste tires or scrap rubber, if not well processed, have been known to be severely harmful to the environment and human health [12]. The traditional way of scrap rubber processing is disposal by incineration or landfill. However, the ashes emitted into the air from rubber combustion in the incineration plant contribute to air pollution. The inorganic residuals produced by incinerators are sent to landfill space, and they have the risk of being washed out by heavy rain and seep into mud or groundwater. As complementary to producing green products, which are ideally zero-waste and environmentally friendly, waste management and recycling technology are direct solutions to the minimization of waste and end-of-life product disposals for the current production model [5]. More efficient ways for rubber recycling have been developed. For instance, tire pyrolysis involves thermal degradation of waste tires and produces tire pyrolysis oil, pyro char, and pyro gas. Tire pyrolysis oil can be used for alternative engine fuel. Pyro char can be used as energy storage material, and pyro gas can be used as industrial fuel [13]. The rubber 
granulate processed from waste has been used in practice to produce floor coverings, cow mattresses, and stand systems for signposting of road works and barriers [14]. This closed circular economy for rubber not only reduces the carbon footprint but also solves the waste processing problem of scrap tires and rubber.

The quantity of scrap rubber dumped by Taiwan rubber industries nearly doubled in the last decade, from 3.486 million metric tons (MT) in 2011 to 6.14 MT in 2019 [15]. However, the capacity of scrap rubber incineration and landfill only increased by about $25 \%$ during the same period, necessitating a large temporary storage space for scrap rubber. It is estimated that $57 \%$ of the 6.14 million tons of scrap rubber in 2019 can be actually reused or recycled and should not be disposed by incinerators and landfill, indicating there is much room to redesign closed circular materials for rubber industries in Taiwan.

In this paper we study one of the leading rubber manufacturers in Taiwan, which aims at circular rubber material designs. The manufacturer has two main production lines. The first line produces rolling cylinders, rubber rollers, sander wheel, and packing rubber for use by many different industries. The second line reuses the scrap rubber granulates and strips produced by the first line and mixes them with other materials to manufacture light-weight rubber works such as construction materials, barriers, and stand systems for signposting of road works. A typical process of the production is shown in Figure 1. The rubber materials go through several operations including compounding, coating, molding, vulcanization, demolding, and deflashing. During each operation, certain quantities of scrap rubber are yielded, which can be reused to make light-weight rubber products. Some of the products produced by the two lines are shown in Figure 1g,h. An application of the circular rubber products, the stone-like wall rubber, is shown in Figure 1i.

The current practice of the two production lines follows a linear fashion, i.e., the scheduling for the two production lines is independent. The scrap rubber granulates and strips produced by the first line are put aside in a buffer storage, and the machines in the second line have no expectation for the real-time availability of the circular rubber materials. Hence, the second line is started only when the amount of scrap rubber granulates and strips exceeds the total demand of all the jobs to be processed in the second line. Specifically, if the scheduling of the two production lines is properly aligned, the makespan will be significantly reduced. The objective of this paper is to find the best job sequence for each line such that the maximum completion time for all jobs on both lines is as short as possible. Hereafter, we name the addressed problem as the circular rubber manufacturing problem (CRMP). Each production line in the CRMP is a flowshop where a number of jobs should be processed by the same sequence of operations. As the flowshop scheduling problem is NP-Hard [16], a polynomial-time exact algorithm does not exist unless P = NP. Many approximation algorithms have been proposed. Early methods consider machines as the only resource in the flowshop and propose effective heuristics. Johnson [17] proposed an $n$-job, two-machine exact algorithm, which has been widely embedded in later heuristics for solving general $n$-job, $m$-machine flowshop problems. One of the notable approximation algorithms is the Campbell-Dudek heuristic [18], which considers the first $k$ machines as machine $\mathrm{A}$ and the last $k$ machines as machine $\mathrm{B}$, then solves the resulting $n$-job, twomachine problem by applying Johnson's algorithm to obtain the optimal job sequencing. The process is repeated through $k=1, \ldots, m-1$ (so machine A and machine B may cover some original machines in common), and the minimum total processing time among these $m-1$ job sequences is the final approximation solution to the original problem. Instead of formatting the $n$-job, $m$-machine flowshop to multiple $n$-job, two-machine problems, Nawaz et al. [19] presented a NEH heuristic that generates the final job sequence in a constructive way, where the unprocessed job with the longest processing time is tentatively placed at every sequence position, and the best position for the job is evaluated. In this fashion, a new job is incrementally added to the partial sequence at each evaluation step until a full sequence is obtained. In practical flowshop production, multiple types of resources in addition to machines usually exist and need to be properly managed. These resources include raw materials, manpower, budgets, or circular materials, making the 
job scheduling more perplexing. The context of multiple resources constraints is more practical and realistic in manufacturing industries. Laribi et al. [20] deals with makespan minimization in flow shop scheduling problems where a second resource other than machines is considered. By adapting the NEH heuristic for accommodating a second resource constraint, an approximation solution for the $n$-job, $m$-machine flowshop problem with resource constraint can be quickly produced.

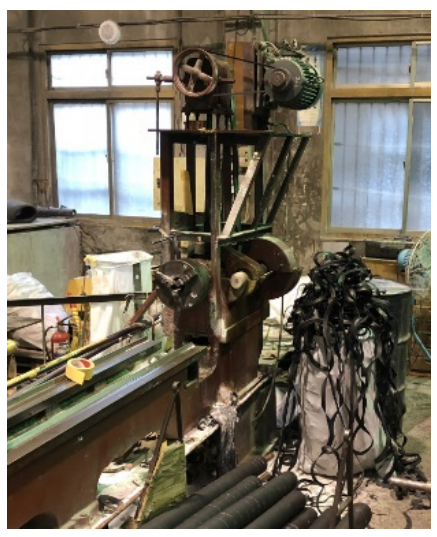

(a)

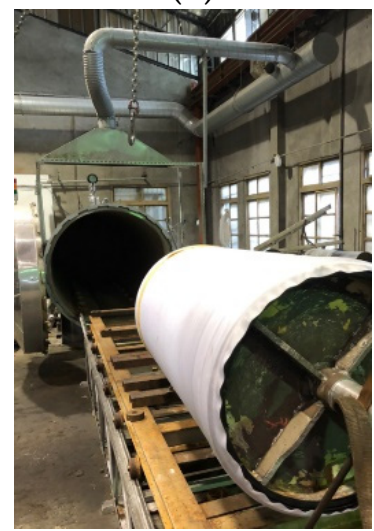

(d)

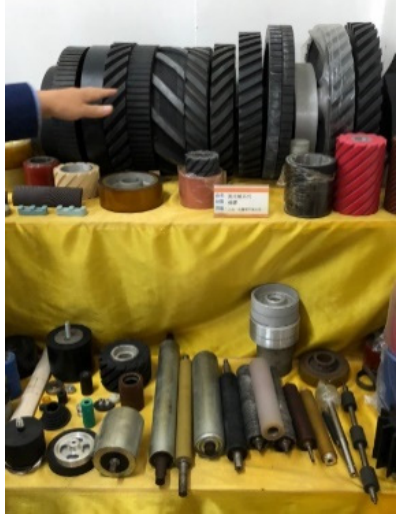

(g)

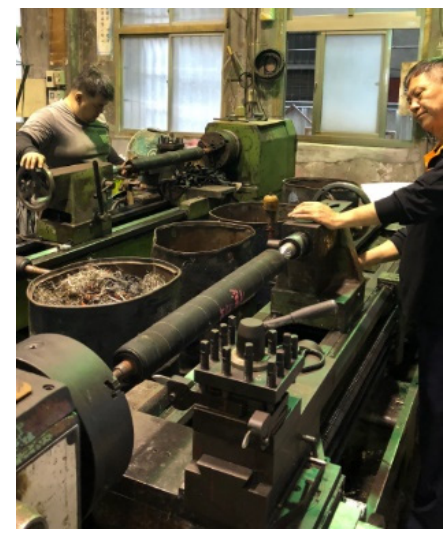

(b)

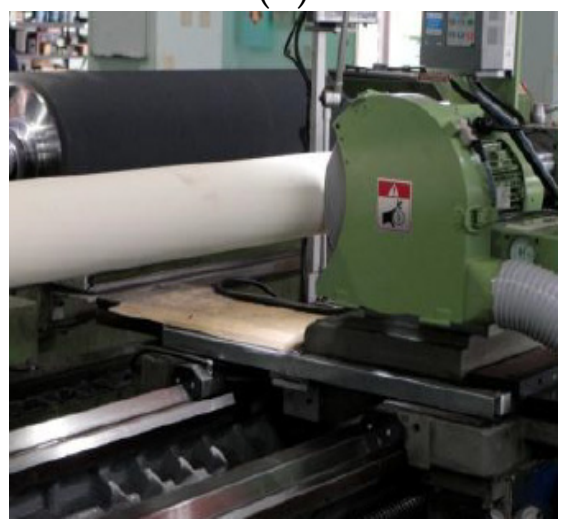

(e)

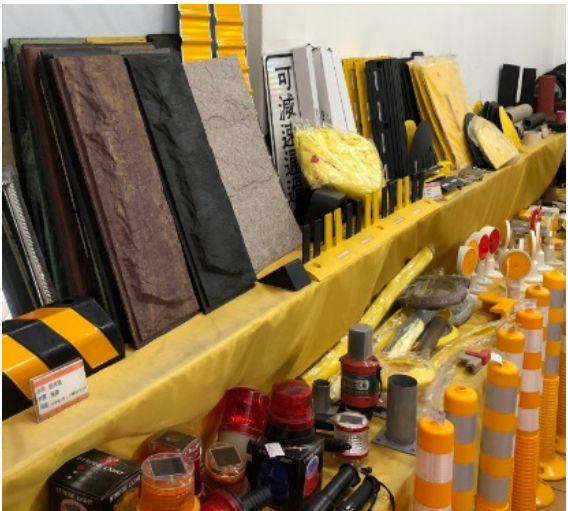

(h)

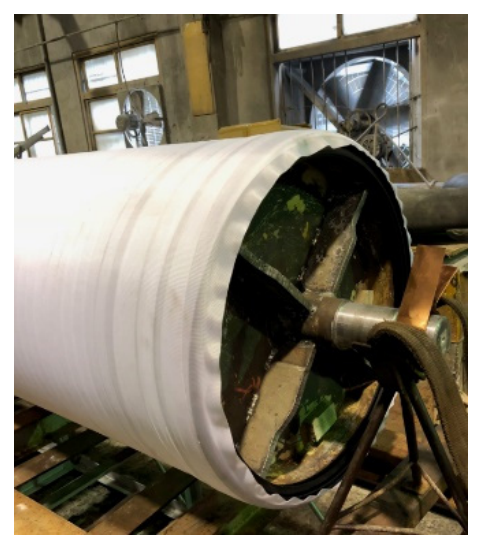

(c)

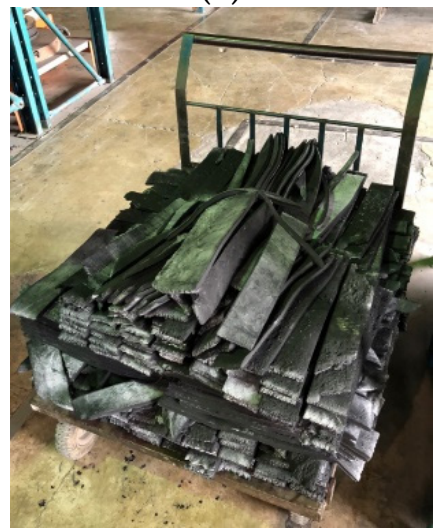

(f)

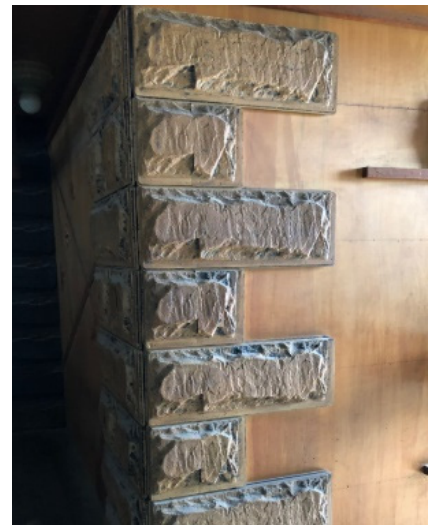

(i)

Figure 1. The typical operations for the production in the rubber manufacturer and exemplar rubber products. (a) Compounding; (b) Coating; (c) Molding; (d) Vulcanization; (e) Demolding; (f) Deflashing; (g) Exemplar products of the first line; (h) Exemplar circular products of the second line; (i) Stone-like wall rubber.

Recently, evolutionary algorithms have shown superior job-sequencing solutions than those obtained by applying heuristics. Osman and Potts [21] designed a simulated anneal- 
ing (SA) algorithm to obtain approximate solutions of the optimal job order to minimize the maximum completion time. A genetic algorithm (GA) is developed by Reeves [22] for finding the approximate minimum makespan of the $n$-job, $m$-machine flowshop problem. It is shown that the performance of the GA surpasses that of a neighborhood search heuristic and an SA. In order to further improve the performance, Murata et al. [23] developed a hybridization of a GA and an SA to achieve a better job sequencing than that obtained by either GA or SA alone. Another hybridization approach was proposed in Eksioglu and Eksioglu [24], which combines the tabu search and a variable neighborhood search heuristic for the flowshop problem.

It is intuitive to apply existing algorithms for the flowshop scheduling to the addressed CRMP problem. However, the CRMP problem is more complex than the flowshop scheduling in that each production line in the CRMP is already a flowshop, and that there are multiple types of circular resources that bind the two production lines to each other. As a result, the research objectives of this study are as follows.

- As our study scheduling context of two-line circular manufacturing has not been contemplated before, a precise mathematical formulation that clearly defines the objectives and the constraints of the problem has to be presented.

- Previous evolutionary algorithms for job-sequencing problems are not directly applicable to the addressed CRMP problem. Therefore, the developed solution method should take the CRMP properties into account and be customized to the problem context to enhance the effectiveness and efficiency.

- The CRMP problem is inspired by the real circular rubber production context, so the experimental design should include the empirical results with the dataset collected from the real production lines of the research subject. Synthetic datasets should also be tested to validate the robustness of the proposed approach.

- The proposed approach needs to be compared to modified versions of the traditional scheduling heuristics and demonstrate its advantages, such that the need for developing a new method customized to CRMP is justified.

From the literature, we learned evolutionary algorithms are effective in finding approximation solutions to the flowshop scheduling. In this paper, a customized GA is developed to tackle the NP hardness of the CRMP. The contributions of our paper are as follows. (1) The traditional process of disposed rubber waste has the risk of poisoning the environment, as indicated in [11,12]. We propose a sustainable rubber circular manufacturing model to reduce rubber waste and save material cost. (2) Exiting scheduling heuristics [17-19] in the literature considers only a single production line and one type of resource. They cannot be directly applied to efficiently tackle the CRMP problem. To achieve efficient circular manufacturing, two production lines of the CRMP must be appropriately aligned according to the reused material constraints. Our method aims at minimizing the makespan of the entire production by elaborating the yielding and reuse of the circular materials. (3) In the literature, evolutionary algorithms [21-24] have shown excellent scheduling capability for flowshop sequencing problems. However, the customized evolutionary algorithms for CRMP are lacking. In this context, we first present a mathematical formulation for the newly introduced CRMP problem and then develop a GA that is customized to the specifications of the CRMP. Our GA design guarantees the reproduced chromosomes are feasible solutions to the CRMP problem, making the evolution efficient without the need for handling infeasible solutions. (4) Our studied subject is a leading rubber manufacturer. We cooperatively developed the two-line circular manufacturing model. The real data collected from the daily operations in the factory are used in the experiment. To evaluate the robustness of our GA, a comprehensive dataset that synthesizes the scenarios in manufacturing is further tested. The experimental result shows that the proposed GA outperforms two heuristics in shortening their makespan by $3.4-9.7 \%$ and $3.5-11.2 \%$ for the real and synthetic datasets, respectively. 
The remainder of the paper is organized as follows. Section 2 presents the problem statements and the proposed methods. Section 3 elucidates the experimental results and comparative performances. Section 4 concludes this work.

\section{Problem Statements and Proposed Methods}

\subsection{Notations}

$$
\begin{aligned}
& \text { Decision variables } \\
& x_{l, i_{1}, i_{2}}= \begin{cases}1, & \text { if job } i_{1} \text { operation precedes job } i_{2} \text { operation on line } l, l \in\{\mathrm{A}, \mathrm{B}\} \\
0, & \text { otherwise. }\end{cases} \\
& y_{i_{1}, j_{1}, i_{2}, j_{2}}=\left\{\begin{array}{rr}
1, & \text { if job } i_{1} \text { operation on machine } j_{1} \text { of Line A is completed before } \\
0, & \text { the starting of job } i_{2} \text { operation on machine } j_{2} \text { of Line B }
\end{array}\right.
\end{aligned}
$$

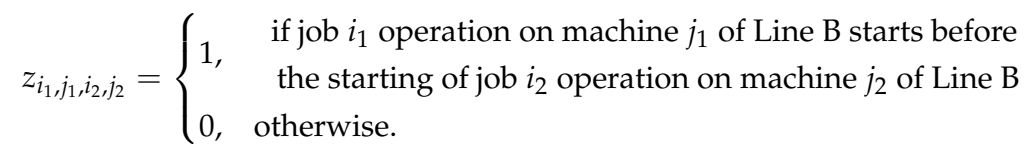

$$
\begin{aligned}
& \text { Parameters } \\
& C_{\text {max }} \\
& C_{l, i, j} \\
& p_{l, i, j} \\
& r_{i, j, k} \\
& d_{i, j, k}
\end{aligned}
$$

\subsection{Problem Statements and Formulation}

We consider the practical case of a rubber manufacturer in Taiwan. The addressed subject has two production lines, denoted line A and line B, respectively. There are $n_{1}$ jobs to be processed on line $\mathrm{A}$, and all jobs on this line go through the same $m_{1}$ operations, which should be performed in a specific order. All of the first operations of these jobs are processed on the first machine, all of the second operations on the second, etc. Each job is processed by only one operation at a time, and no preemption is allowed. The flow control implies that the processed job sequence on each machine is the same. Similar flow control is applied to line B with $n_{2}$ jobs and $m_{2}$ operations. Each job operation upon finishing on line A will yield two types of circular materials (scrap rubber granulates and strips), which can be reused or mixed with other materials in the job operations on line B. The start of job operation on line B should wait until all required circular materials are received from the yielding of line A. The aim of our circular rubber manufacturing problem (CRMP) is to determine the optimal flowshop schedules for the two lines such that the makespan of all jobs is a minimum. The CRMP is NP-Hard given that each individual line of CRMP is itself a flowshop scheduling problem, which has been known as NP-Hard when there are more than two machines [16].

Before presenting the problem formulation, an illustrative example is given to elucidate the CRMP. Assume line A has three jobs $\left\{J_{1}^{\mathrm{A}}, J_{2}^{\mathrm{A}}, J_{3}^{\mathrm{A}}\right\}$ and two machines $\left\{M_{1}^{\mathrm{A}}, M_{2}^{\mathrm{A}}\right\}$, line $\mathrm{B}$ also has three jobs $\left\{J_{1}^{\mathrm{B}}, J_{2}^{\mathrm{B}}, J_{3}^{\mathrm{B}}\right\}$ and two machines $\left\{M_{1}^{\mathrm{B}}, M_{2}^{\mathrm{B}}\right\}$. The processing time of each job on each operation of line $A$ and the quantities of yielded scrap rubbergranulates and strips from each operation are given in Table 1. Similar descriptive data (yielded materials become the required circular materials) for line $\mathrm{B}$ are displayed in Table 2. Figure 2 shows feasible job schedules, $\left\{J_{1}^{\mathrm{A}}, J_{3}^{\mathrm{A}}, J_{2}^{\mathrm{A}}\right\}$ for line $\mathrm{A}$ and $\left\{J_{3}^{\mathrm{B}}, J_{2}^{\mathrm{B}}, J_{1}^{\mathrm{B}}\right\}$ for line $\mathrm{B}$. The obtained makespan is 21 . 
Table 1. The processing time and yielded materials of each job on each operation of line A.

\begin{tabular}{cccc}
\hline & $J_{1}^{\mathbf{A}}$ & $J_{2}^{\mathbf{A}}$ & $J_{3}^{\mathbf{A}}$ \\
\hline$M_{1}^{\mathrm{A}}$ & $4,3,1$ & $5,1,3$ & $3,2,2$ \\
$M_{2}^{\mathrm{A}}$ & $5,3,2$ & $2,2,2$ & $6,1,1$ \\
\hline
\end{tabular}

Table 2. The processing time and required circular materials of each job on each operation of line B.

\begin{tabular}{cccc}
\hline & $J_{1}^{\mathbf{A}}$ & $J_{2}^{\mathbf{A}}$ & $J_{3}^{\mathbf{A}}$ \\
\hline$M_{1}^{\mathrm{A}}$ & $2,0,1$ & $3,2,2$ & $4,7,1$ \\
$M_{2}^{\mathrm{A}}$ & $1,1,0$ & $2,1,1$ & $4,1,5$ \\
\hline
\end{tabular}

job order for line A: $\left\{J_{1}^{\mathrm{A}}, J_{3}^{\mathrm{A}}, J_{2}^{\mathrm{A}}\right\}$

line A $M_{1}^{\mathrm{A}}$ : $\begin{array}{llllllllllllllllllllllll}0 & 1 & 2 & 3 & 4 & 5 & 6 & 7 & 8 & 9 & 10 & 11 & 12 & 13 & 14 & 15 & 16 & 17 & 18 & 19 & 20 & 21 & 22\end{array}$

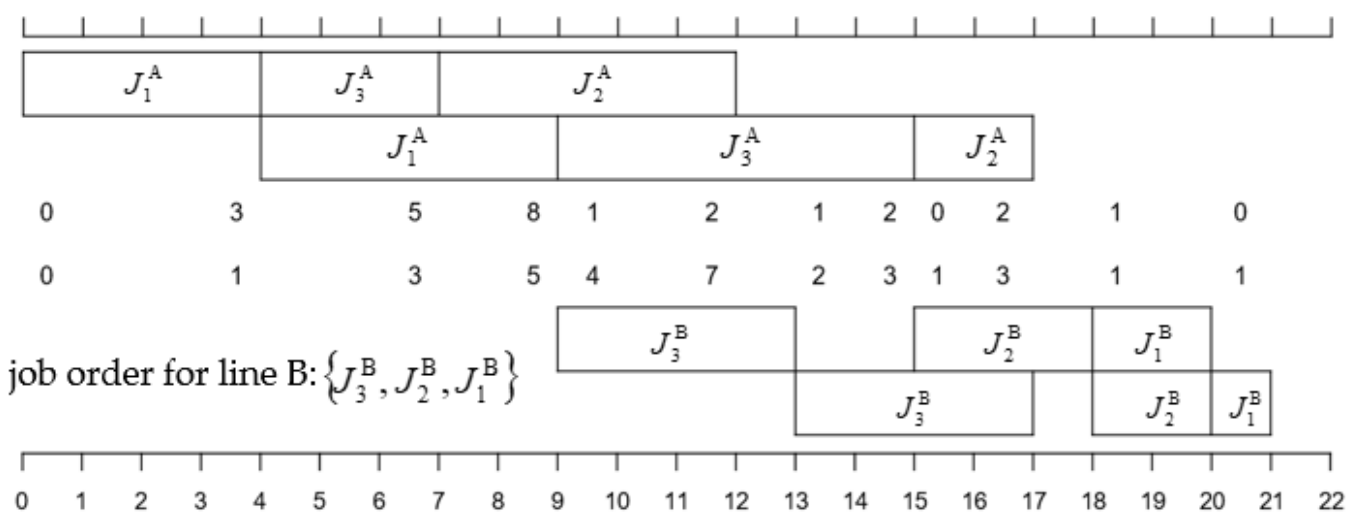

Figure 2. Feasible job schedules for line A and line B under the production specifications given in Tables 1 and 2.

Following the notations and the numerical example introduced, we now present the integer programming formulation of CRMP as follows.

$$
\text { Minimize } C_{\max }
$$

Subject to:

$$
\begin{aligned}
& C_{\max }>C_{l, i, j} \forall i, \forall j, \forall l ; \\
& C_{l, i, j_{2}} \geq C_{l, i, j_{1}}+p_{l, i, j_{2}} \forall i, \forall j_{1}<j_{2} \forall l ; \\
& C_{l, i_{2}, j} \geq C_{l, i_{1}, j} x_{l, i_{1}, i_{2}}+p_{l, i_{2}, j} \forall i_{1} \neq i_{2}, \forall j \forall l \text {; } \\
& x_{l, i_{1}, i_{2}}+x_{l, i_{2}, i_{1}}=1 \quad \forall i_{1} \neq i_{2}, \forall l ; \\
& x_{l, i_{1}, i_{2}}+x_{l, i_{2}, i_{3}}+x_{l, i_{3}, i_{1}} \leq 2 \forall i_{1} \neq i_{2} \neq i_{3}, \forall l ; \\
& d_{i_{2}, j_{2}, k} \leq \sum_{\forall i_{1}} \sum_{\forall j_{1}} r_{i_{1}, j_{1}, k} y_{i_{1}, j_{1}, i_{2}, j_{2}}-\sum_{\forall i_{3}} \sum_{\forall j_{3}} d_{i_{3}, j_{3}, k} z_{i_{3}, j_{3}, i_{2}, j_{2}} \quad \forall i_{2}, \quad \forall j_{2}, \forall k ; \\
& y_{i_{1}, j_{1}, i_{2}, j_{2}} C_{\mathrm{A}, i_{1}, j_{1}} \leq y_{i_{1}, j_{1}, i_{2}, j_{2}}\left(C_{\mathrm{B}, i_{2}, j_{2}}-p_{\mathrm{B}, i_{2}, j_{2}}\right) \forall i_{1}, \forall j_{1}, \forall i_{2}, \forall j_{2} ; \\
& \left(1-y_{i_{1}, j_{1}, i_{2}, j_{2}}\right) C_{\mathrm{A}, i_{1}, j_{1}} \geq\left(1-y_{i_{1}, j_{1}, i_{2}, j_{2}}\right)\left(C_{\mathrm{B}, i_{2}, j_{2}}-p_{\mathrm{B}, i_{2}, j_{2}}\right) \forall i_{1}, \forall j_{1}, \forall i_{2}, \forall j_{2} ; \\
& z_{i_{1}, j_{1}, i_{2}, j_{2}}\left(C_{\mathrm{B}, i_{1}, j_{1}}-p_{\mathrm{B}, i_{1}, j_{1}}\right) \leq z_{i_{1}, j_{1}, i_{2}, j_{2}}\left(C_{\mathrm{B}, i_{2}, j_{2}}-p_{\mathrm{B}, i_{2}, j_{2}}\right) \forall i_{1}, \forall j_{1}, \forall i_{2}, \forall j_{2} ; \\
& \left(1-z_{i_{1}, j_{1}, i_{2}, j_{2}}\right)\left(C_{\mathrm{B}, i_{1}, j_{1}}-p_{\mathrm{B}, i_{1}, j_{1}}\right) \geq\left(1-z_{i_{1}, j_{1}, i_{2}, j_{2}}\right)\left(C_{\mathrm{B}, i_{2}, j_{2}}-p_{\mathrm{B}, i_{2}, j_{2}}\right) \forall i_{1}, \forall j_{1}, \forall i_{2}, \forall j_{2} ; \\
& x_{l, i_{1}, i_{2}} \in\{0,1\} \forall i_{1} \neq i_{2}, \forall l ; \\
& y_{i_{1}, j_{1}, i_{2}, j_{2}} \in\{0,1\} \forall i_{1}, \forall j_{1}, \forall i_{2}, \forall j_{2} ;
\end{aligned}
$$




$$
z_{i_{1}, j_{1}, i_{2}, j_{2}} \in\{0,1\} \forall i_{1}, \forall j_{1}, \forall i_{2}, \forall j_{2}
$$

Equation (1) is the objection function corresponding to the minimization of the makespan of the entire production consisting of Line A and Line B. Constraint (2) stipulates the makespan should be greater than the completion time of any job on any machine. Constraint (3) states that the operation of job $i$ on machine $j_{2}$ can only start after its operation on any preceding machines has completed, so the completion time of job $i$ on machine $j_{2}$ should be larger than the sum of its processing time and the completion time of any preceding machine $j_{1}$ in the same production line. Constraint (4) entails that the operation of any two distinct jobs on the same machine (job $i_{1}$ operation precedes job $i_{2}$ operation on machine $j$ ) cannot be preempted. Equations (5) and (6) guarantee the job flow constraints. Constraint (7) calls for the satisfaction of the circular material demand for processing job $i_{2}$ on machine $j_{2}$ in Line B by requiring the demand is less than or equal to all the materials already yielded in Line A minus all the consumed materials in Line B. Equations (8)-(11) are used to validate the decision variables $y_{i_{1}, j_{1}, i_{2}, j_{2}}$ and $z_{i_{1}, j_{1}, i_{2}, j_{2}}$, while Equations (12)-(14) claim that all decision variables are binary ones. The integer programming model of small problem sizes can be solved to optimality by using commercial software such as lingo. However, for medium- and large-sized problems, quality solutions may be obtained by applying evolutionary algorithms such as GAs.

The problem modeling with our formulation is limited to the research subject and our interactions with the rubber manufacturer. It may not reflect the production context of other rubber manufacturers, which has potential to implement circular manufacturing.

\subsection{Proposed Methods}

GAs are bio-inspired natural algorithms that simulate Darwinian evolution theory to improve the performance of computational systems. GAs have been used for several decades in applications such as machine learning [25], engineering optimization [26], and operational management [27], to name a few. It has been proven in Holland's schemata theory [28] that GAs exponentially converge to the global optimum if no dependency exists among the genes. In general, GAs manipulate evolution as follows. The solution structure to the addressed problem is encoded as a chromosome, which is usually a string of numbers. A fitness function of a chromosome is defined to evaluate how fit the delivered gene values by the chromosome solve the problem at hand. An initial population consisting of many chromosome instances is created by random. The population evolves through successive generations by repeatedly performing natural genetic operations, namely selection, crossover, and mutation. When the evolution terminates with a maximum number of generations, the chromosome with the best fitness value observed so far is output as the final solution. In the following, we articulate our GA design customized to the CRMP problem.

Assuming that there are $n_{1}$ jobs $\left\{J_{1}^{\mathrm{A}}, J_{2}^{\mathrm{A}}, \ldots, J_{n_{1}}^{\mathrm{A}}\right\}$ to be processed by line $\mathrm{A}$ and $n_{2}$ jobs $\left\{J_{1}^{\mathrm{B}}, J_{2}^{\mathrm{B}}, \ldots, J_{n_{2}}^{\mathrm{B}}\right\}$ by line B. A feasible job processing schedule to the CRMP problem can be represented by a chromosome integer string as $\mathrm{X}={ }^{\prime} x_{1}, x_{2}, \ldots, x_{n_{1}+n_{2}}{ }^{\prime}$ where $1 \leq x_{i} \leq$ $n_{1}+n_{2}$ and $x_{i} \neq x_{j}$ if $i \neq j$. In other words, $\mathrm{X}$ is an integer permutation where $x_{i}$ indicates $J_{i}^{\mathrm{A}}$ if $1 \leq x_{i} \leq n_{1}$ and $x_{i}$ indicates $J_{i-n_{1}}^{\mathrm{B}}$ if $n_{1}+1 \leq x_{i} \leq n_{1}+n_{2}$. For example, let there be 4 and 3 jobs to be processed by line $\mathrm{A}$ and line $\mathrm{B}$, respectively. A chromosome $\mathrm{X}={ }^{\prime} 3614725^{\prime}$ indicates the job order $\left\{J_{3}^{\mathrm{A}}, J_{1}^{\mathrm{A}}, J_{4}^{\mathrm{A}}, J_{2}^{\mathrm{A}}\right\}$ for line $\mathrm{A}$ and $\left\{J_{2}^{\mathrm{B}}, J_{3}^{\mathrm{B}}, J_{1}^{\mathrm{B}}\right\}$ for line $\mathrm{B}$. The fitness value of the chromosome is evaluated by calculating the makespan with the job orders contained in the chromosome. In this context, the lower the fitness value (makespan) is, the better the chromosome.

An initial population $\mathbf{P}$ of $N$ chromosomes is generated at random to start the evolution. The fitness value for each chromosome in $\mathbf{P}$ is evaluated. Natural selection is a process that mimics the survival of the fitter chromosomes. We adopt the tournament selection scheme to generate the next population $\mathbf{Q}$ from the current population $\mathbf{P}$. The selection process is as follows. For each empty slot in the next population $\mathbf{Q}$, two chromosomes are randomly selected from $\mathbf{P}$ to compete for survival. The winner of the competition is 
determined by reference to the fitness values, and the winner is cloned to fill the empty slot. Both competitors still remain in $\mathbf{P}$ and are eligible for selection in the next round. The selection process is repeated until all the empty slots in the next population $\mathbf{Q}$ are filled up. The tournament selection is superior to earlier selection schemes like roulette-wheel selection, which has a high risk of generating large stochastic errors.

The crossover operation manipulates two parental chromosomes and exchange genes between them to produce offspring chromosomes. The crossover operation is performed with a crossover probability $P_{c}$. In other words, $N \times P_{c}$ chromosomes will be manipulated by crossover. As our chromosome structure is represented as a permutation string, the partially matched crossover (PMX) that can reorder the permutation is applied. The PMX crossover resembles the two-point crossover but respects a look-up table to avoid repetitions of gene alleles. The PMX crossover consists of three steps: look-up table establishment, gene exchange, and gene correction. An example is illustrated in Figure 3 where two parental chromosomes $X$ and $Y$ are shown with the PMX crossover to produce two offspring chromosomes $X^{\prime}$ and $Y^{\prime}$. Firstly, two cutting sites are generated at random, say at 3 and 6 . The gene segments between the two cutting sites are used to establish a lookup table, i.e., the list $(2,3,10)$ is mapped to $(5,6,7)$. Secondly, the gene segments between the two cutting sites are exchanged. Finally, the repetitive genes in the remaining segments due to the exchange are corrected by referring to the look-up table (the corresponding genes before and after correction are printed in red as shown in Figure 3).
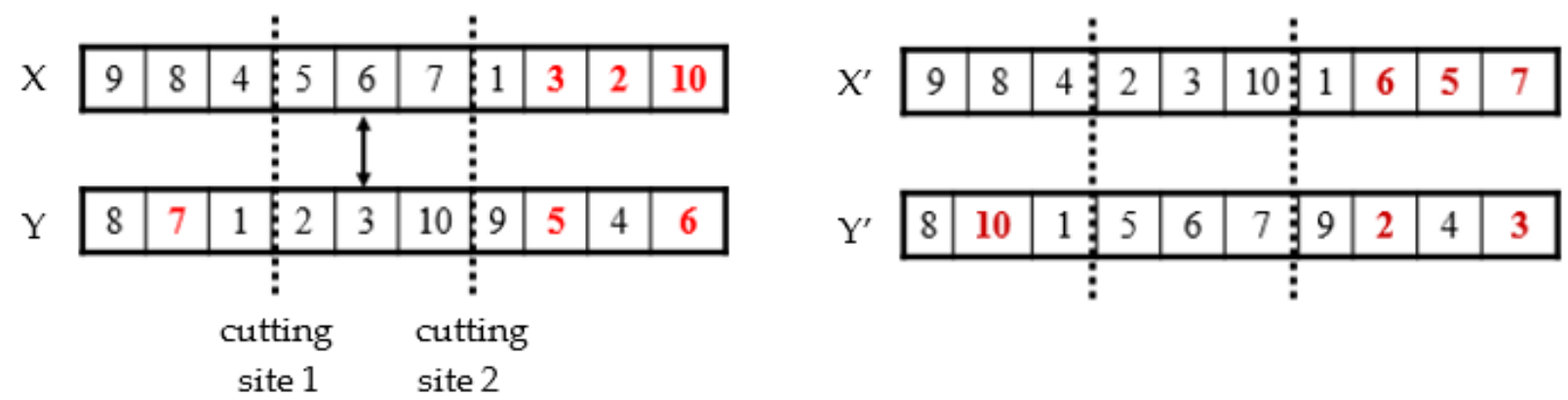

Figure 3. Two parental chromosomes $X$ and $Y$ are shown with the PMX crossover to produce two offspring chromosomes $X^{\prime}$ and $Y^{\prime}$.

Mutation alters the gene alleles of a chromosome and is performed with a mutation probability $P_{m}$. Mutation is an imperative operation to reserve the gene diversity in the population in order to avoid premature convergence. The ordinary flipping mutation, which mutates the gene by replacing its allele with an alternative one, is not applicable to our permutation-based chromosomes because there is risk of generating repetitive gene alleles in a chromosome. Here, we employ the 2-swap mutation that randomly selects two genes and exchanges them. The 2-swap operation is a common heuristic that has been embedded in sophisticated methods for combinatorial optimization problems.

In summary, the pseudo code of our GA for the CRMP problem is shown in Figure 4. The evolution will be executed until a maximal number of generations have been reproduced. Upon the end of the evolution, the job sequences contained in the best chromosome observed in the entire evolution are used to solve the CRMP problem. 
1 Initialization step:

1.1 Randomly generate a population $\mathbf{P}$ of $N$ chromosomes. Each chromosome is a permutation of the set of natural numbers $\left\{1,2, \ldots, n_{1}+n_{2}\right\}$

1.2 Evaluate fitness value for each chromosome in $\mathbf{P}$

2 Evolution step: repeat until the maximal number of evolutionary generation is met 2.1 Tournament Selection:

2.1.1 For each empty slot in $\mathbf{Q}$, randomly choose two chromosomes from $\mathbf{P}$ to compete. The winner is cloned to fill up the empty slot in $\mathbf{Q}$

\subsection{2 $\quad \mathbf{P} \leftarrow \mathbf{Q}$}

2.1.3 Set $\mathbf{Q}$ to empty

\subsection{PMX Crossover:}

2.2.1 Randomly choose two chromosomes in $\mathbf{P}$

2.2.2 Perform PMX crossover with the crossover probability $P_{c}$

2.3 2-Swap Mutation: For each chromosome, perform the 2-swap mutation with the mutation probability $P_{m}$

2.4 Evaluate fitness value for each chromosome in $\mathbf{P}$

3 Output the job sequences contained in the best chromosome

Figure 4. The pseudo code of our GA for the CRMP problem.

\section{Results}

The CRMP problem was formulated from the real context of circular rubber products manufacturing. A real dataset was provided by the investigated manufacturer. To further testify the characteristics of our GA, a comprehensive synthetic dataset was generated to cover diverse profiles of production. The computation platform for our experiments was a personal computer with a $2.4 \mathrm{GHz}$ CPU and $8 \mathrm{~GB}$ RAM, and the programs were codified using Python 3.8.5 and C\#.

\subsection{Experiment with Real Dataset}

Our study subject was a rubber manufacturer in Taiwan that provided us real data collected from the productions. We experimented with two production lines. The first line (denoted Line A) consisted of six machines and was the line for producing main rubber products. The second line (denoted Line B) had three machines, and it was where the lightweight rubber works are manufactured. Following the notations defined in Section 2.1, the parameters of both production lines in the real data are shown in Tables 3 and 4, respectively. We compared our GA with two scheduling heuristics. The First-Come-First-Serve heuristic (denoted by FCFS) processes the jobs in the order with which the jobs come to the line. The Campbell-Dudek heuristic [18] as previously introduced in Section 1 iteratively applies Johnson's algorithm $m-1$ times for the $n$-job, $m$-machine problem, and the best schedule of the $m-1$ job sequences is returned as the near-optimal scheduling. As the CRMP problem has two production lines, the Campbell-Dudek heuristic should be separately applied to each line, and the best schedule of all possible combined job sequences from both lines is returned. The parameter setting of our GA is as follows. The population consisted of 20 chromosomes that evolve for 50 generations. During each generation, the next population was generated by applying the tournament selection, PMX crossover with probability 0.8 , and the 2 -swap mutation with probability 0.1 . The best chromosome (job sequence) obtained in the entire evolution was returned. As GA is a stochastic optimization method, each single run of it may produce a distinct best solution from the evolution. To provide a reliable estimation, the GA was executed for 30 independent runs, and the descriptive statistics will be reported. 
Table 3. The processing time and yielded materials for line A.

\begin{tabular}{ccccccccc}
\hline & $J_{1}^{\mathbf{A}}$ & $J_{2}^{\mathbf{A}}$ & $J_{3}^{\mathbf{A}}$ & $J_{4}^{\mathbf{A}}$ & $J_{5}^{\mathbf{A}}$ & $J_{6}^{\mathbf{A}}$ & $J_{7}^{\mathbf{A}}$ \\
\hline$M_{1}^{\mathrm{A}}$ & $115,63,15$ & $77,74,35$ & $107,96,5$ & $93,140,54$ & $91,74,49$ & $62,12,28$ & $77,28,38$ & $72,46,40$ \\
$M_{2}^{\mathrm{A}}$ & $21,20,13$ & $5,4,1$ & $26,33,5$ & $23,32,13$ & $15,6,4$ & $10,11,6$ & $17,19,5$ & $25,22,3$ \\
$M_{3}^{\mathrm{A}}$ & $10,15,5$ & $14,17,7$ & $14,23,3$ & $11,14,2$ & $10,7,4$ & $14,2,5$ & $11,5,5$ & $14,12,8$ \\
$M_{4}^{\mathrm{A}}$ & $173,147,37$ & $113,122,66$ & $132,57,59$ & $169,141,76$ & $92,29,29$ & $145,140,27$ & $165,107,8$ & $114,150,63$ \\
$M_{5}^{\mathrm{A}}$ & $12,11,6$ & $7,9,2$ & $3,1,1$ & $14,22,4$ & $8,6,2$ & $4,2,2$ & $5,6,2$ & $11,4,6$ \\
$M_{6}^{\mathrm{A}}$ & $52,39,20$ & $111,33,68$ & $36,28,3$ & $107,91,64$ & $53,37,8$ & $68,67,43$ & $50,68,15$ & $66,107,11$ \\
\hline
\end{tabular}

Table 4. The processing time and required circular materials for line B.

\begin{tabular}{ccccccc}
\hline & $J_{1}^{\mathbf{B}}$ & $J_{2}^{\mathbf{B}}$ & $J_{3}^{\mathbf{B}}$ & $J_{4}^{\mathbf{B}}$ & $J_{5}^{\mathbf{B}}$ & $J_{6}^{\mathbf{B}}$ \\
\hline$M_{1}^{\mathrm{B}}$ & $51,134,42$ & $54,101,82$ & $37,88,45$ & $71,75,37$ & $32,127,30$ & $78,218,105$ \\
$M_{2}^{\mathrm{B}}$ & $21,76,18$ & $43,40,40$ & $40,114,21$ & $19,71,24$ & $31,72,25$ & $26,65,41$ \\
$M_{3}^{\mathrm{B}}$ & $84,98,103$ & $75,114,44$ & $110,116,96$ & $85,288,55$ & $96,196,50$ & $112,189,111$ \\
\hline
\end{tabular}

Table 5 summarizes the experimental results obtained by the three competing methods. The FCFS heuristic always produced the increasing-order job sequence as Line $\mathrm{A}=\{1$, $2,3,4,5,6,7,8\}$ and Line $B=\{1,2,3,4,5,6\}$, whose makespan was 1457 min. For the Campbell-Dudek heuristic, 10 combinations of job sequences from both lines were produced, and the best scheduling was Line $A=\{6,2,4,8,5,1,7,3\}$ and Line $B=\{5,3,1,2$, $4,6\}$ with the makespan being $1340 \mathrm{~min}$. The average makespan of the 10 combinations of job sequences was $1361 \mathrm{~min}$ with a standard deviation (Std) of 17.46. The best solution obtained from the 30 GA runs was Line $A=\{6,1,2,7,8,4,5,3\}$ and Line $B=\{1,3,6,5,4$, 2 \}, which gave the prevailing makespan of $1307 \mathrm{~min}$. This was $33 \mathrm{~min}(2.5 \%)$ shorter than the best makespan obtained by the Campbell-Dudek heuristic, and was $150 \mathrm{~min}(10.3 \%)$ shorter than the makespan obtained by the FCFS heuristic The average makespan from the 30 independent runs of GA was $1315 \mathrm{~min}$, which was 3.4\% and $9.7 \%$ shorter than the average makespan obtained by the Campbell-Dudek heuristic and FCFS, respectively. It is seen from the Std value that the variation of produced makespans among multiple GA runs was significantly smaller than that of the multiple job-sequence combinations obtained by the Campbell-Dudek heuristic, indicating that the GA was stable and insensitive to the initial solutions generated by random. The CPU time spent for executing GA was $1.2764 \mathrm{~s}$, which is feasible for most scheduling applications, though it is greater than that required by the two heuristics.

Table 5. The experimental results of the three competing methods with the real dataset.

\begin{tabular}{ccccc}
\hline & Best & Average & Std & CPU Time \\
\hline FCFS & 1457 & 1457 & 0 & $6.7 \times 10^{-6}$ \\
Campbell-Dudek & 1340 & 1361 & 17.46 & 0.0223 \\
GA & 1307 & 1315 & 8.05 & 1.2764 \\
\hline
\end{tabular}

Figure $5 a$ to Figure $5 c$ illustrates the Gantt chart of the best schedule of both lines and the dynamic availability of the two types of circular materials obtained by FCFS, Campbell-Dudek heuristic, and GA, respectively. In Figure 5a, we observe that the last machine $\left(M_{6}^{\mathrm{A}}\right)$ in Line A of FCFS started operation at $331 \mathrm{~min}$, and Line A finished all its operations at $1326 \mathrm{~min}$. As will be noted, the FCFS job sequence in Line A was inferior to those arranged by the other two competing methods in two aspects: the makespan for Line A and the machine idle time in Line B. It is seen in Figure 5a that there was a long idle time on $M_{3}^{\mathrm{B}}$ after finishing $J_{3}^{\mathrm{B}}$ at $843 \mathrm{~min}$ and before start processing $J_{4}^{\mathrm{B}}$ at $1135 \mathrm{~min}$. Clearly, this is the main bottleneck that deterred the operation of $M_{3}^{\mathrm{B}}$ for the subsequent jobs and resulted in the overall makespan of $1457 \mathrm{~min}$. The reason for this long idle time is the low availability of type 1 circular material at $843 \mathrm{~min}$ (194 $\mathrm{kg}$ of type 1 material remaining) and 
the high demand $\left(288 \mathrm{~kg}\right.$ ) of the same material by $J_{4}^{\mathrm{B}}$. The operation of $J_{4}^{\mathrm{B}}$ on $M_{3}^{\mathrm{B}}$ has to wait until the required material demand is satisfied with the released circular materials from the operations in Line A during the idle time. This phenomenon reflects the appropriateness of job scheduling in Line A, which not only depends on minimizing its makespan but also satisfying the material demand of operations in Line B.

For the job sequence arranged by the Campbell-Dudek heuristic, as shown In Figure $5 \mathrm{~b}$, we observe that the last machine $\left(M_{6}^{\mathrm{A}}\right)$ in Line A started operation at $235 \mathrm{~min}$ and finished its processes at $1228 \mathrm{~min}$; both checking time points were earlier than those in the Gantt chart for FCFS. For Line B, we see the operations on $M_{1}^{\mathrm{B}}$ and $M_{2}^{\mathrm{B}}$ were more compact and finished earlier than those as seen for FCFS. However, the compact operations also incurred shortage of circular materials for the production on $M_{3}^{\mathrm{B}}$. The job $J_{5}^{\mathrm{B}}$ finishing its $M_{2}^{\mathrm{B}}$ operation at $294 \mathrm{~min}$ has to wait for $219 \mathrm{~min}$ in order to gain sufficient circular materials for transiting to the $M_{3}^{\mathrm{B}}$ operation at $513 \mathrm{~min}$. Another idle time occurred on $M_{3}^{\mathrm{B}}$ when the operation transits from $J_{2}^{\mathrm{B}}$ at $896 \mathrm{~min}$ to $J_{4}^{\mathrm{B}}$ at $1057 \mathrm{~min}$, also due to the low availability of type 1 circular material (177 kg of type 1 material remaining at $896 \mathrm{~min}$ ) and the high demand $(288 \mathrm{~kg})$ of the same material by $J_{4}^{\mathrm{B}}$.

For Line A production arranged by GA, as shown in Figure 5c, the makespan for each machine was similar to that obtained by the Campbell-Dudek heuristic, showing superiority than FCFS. For the Gantt chart in Line B, it seems GA produced a compromised job sequence that balanced between early start-processing time and prudent reserve of necessary materials for later production of critical jobs. We see $M_{1}^{\mathrm{B}}$ and $M_{2}^{\mathrm{B}}$ compactly finished operations for the first six jobs and then deterred the operations for the last job $\left(J_{2}^{\mathrm{B}}\right)$ in order to reserve the circular materials for the production of $J_{4}^{\mathrm{B}}$ on $M_{3}^{\mathrm{B}}$. It is seen that the production in Line B suggested by GA was relatively parallel to enhance the production efficiency, in contrast to those suggested by FCFS and Campbell-Dudek heuristic where all operations on $M_{1}^{\mathrm{B}}$ and $M_{2}^{\mathrm{B}}$ finished very early and left $M_{3}^{\mathrm{B}}$ to proceed with operations of later jobs without parallelization with the other machines, which is the main reason there was a long makespan.

\subsection{Experiment with Synthetic Dataset}

In Section 3.1, we have shown that our GA outperformed FCFS and Campbell-Dudek heuristic on the real dataset provided by the manufacturer. To carry out a sound performance evaluation that can be generally applied to diverse scenarios of the CRMP problem, intensive experiments with a comprehensive synthetic dataset were conducted. As the CRMP problem originated from the real production of a rubber manufacturing factory, the real data values of the production parameters (processing time, yielding or demand of type 1 materials, yielding or demand of type 2 materials) as listed in Tables 3 and 4 were used as a reference to create a range for drawing parameter sample values. The width of the sample range was set to $20 \%$ of the real data value, which was also the center of this range. We created ten CRMP synthetic problem instances by drawing random parameter values from the sampling ranges. The synthetic dataset generated in this way not only reserves the basic properties of the rubber circular manufacturing from real data but also generates diverse production scenarios. 


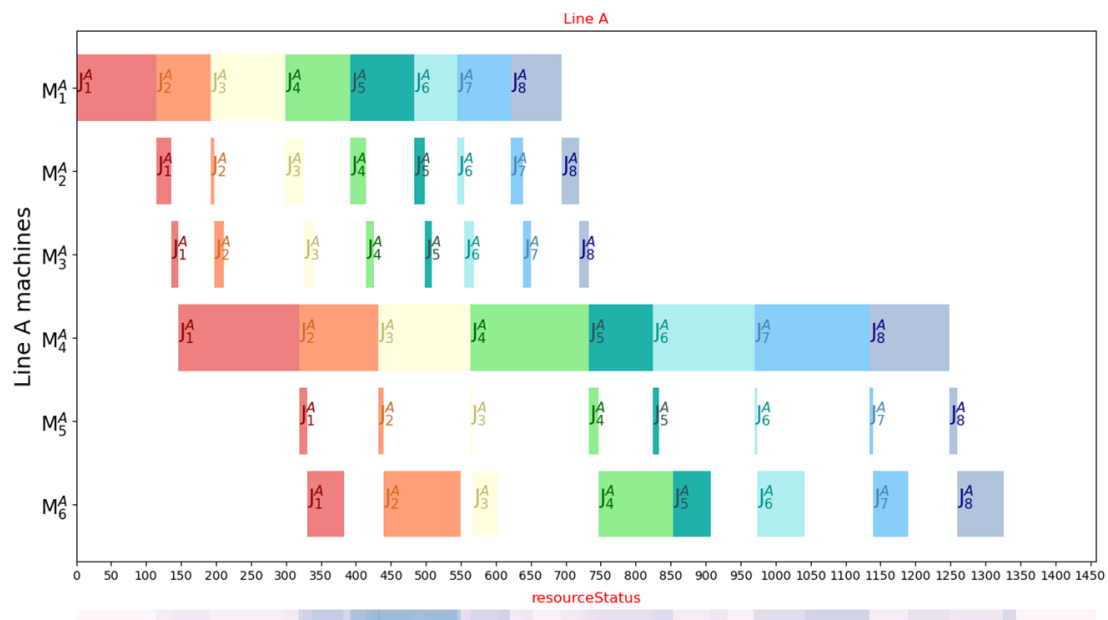

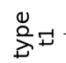

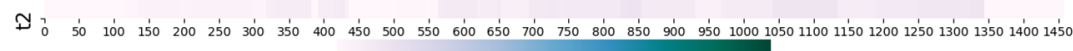

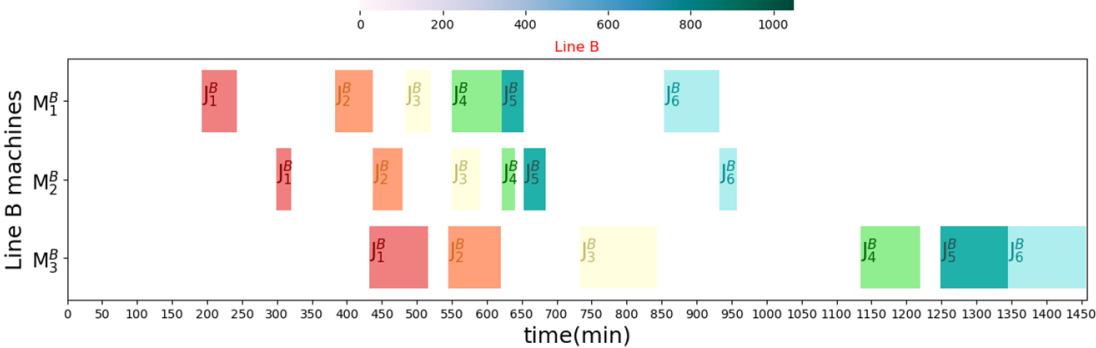

(a)

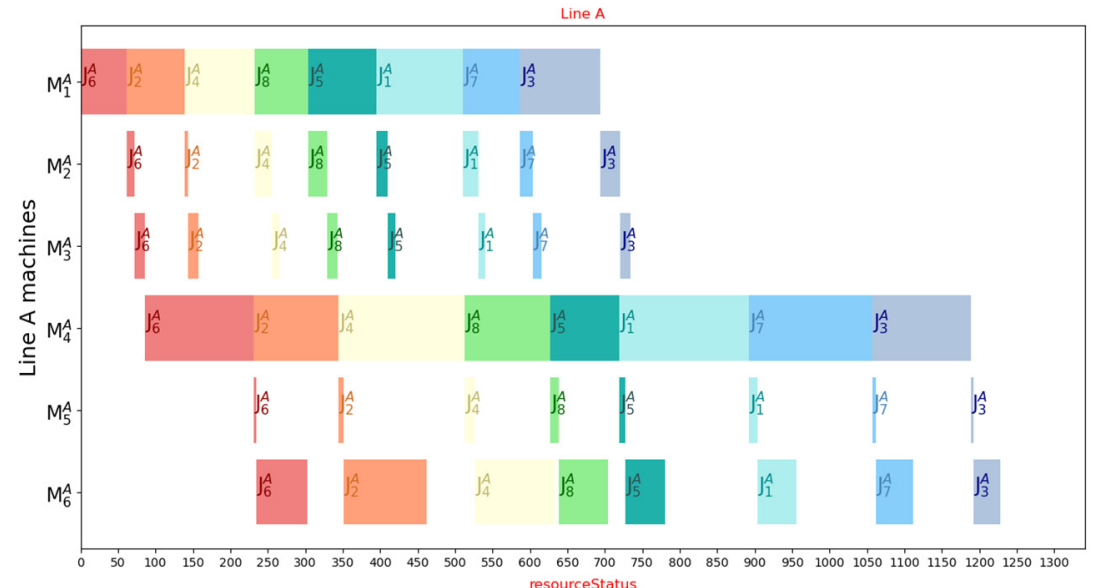

$\stackrel{2}{2}$

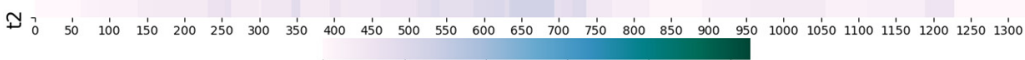

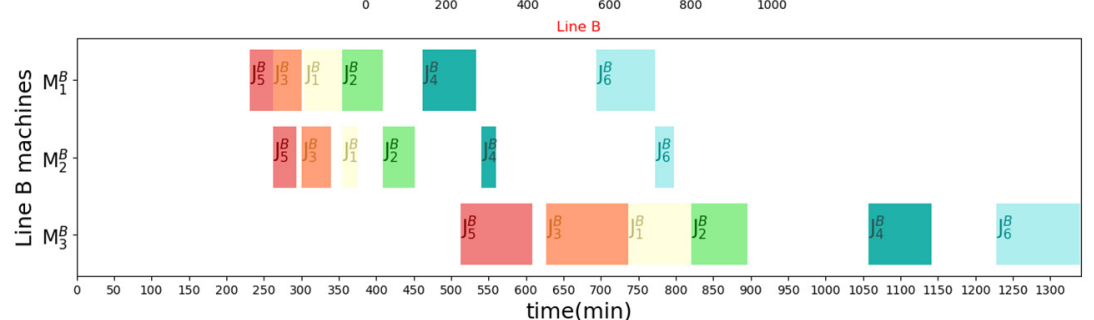

(b)

Figure 5. Cont. 

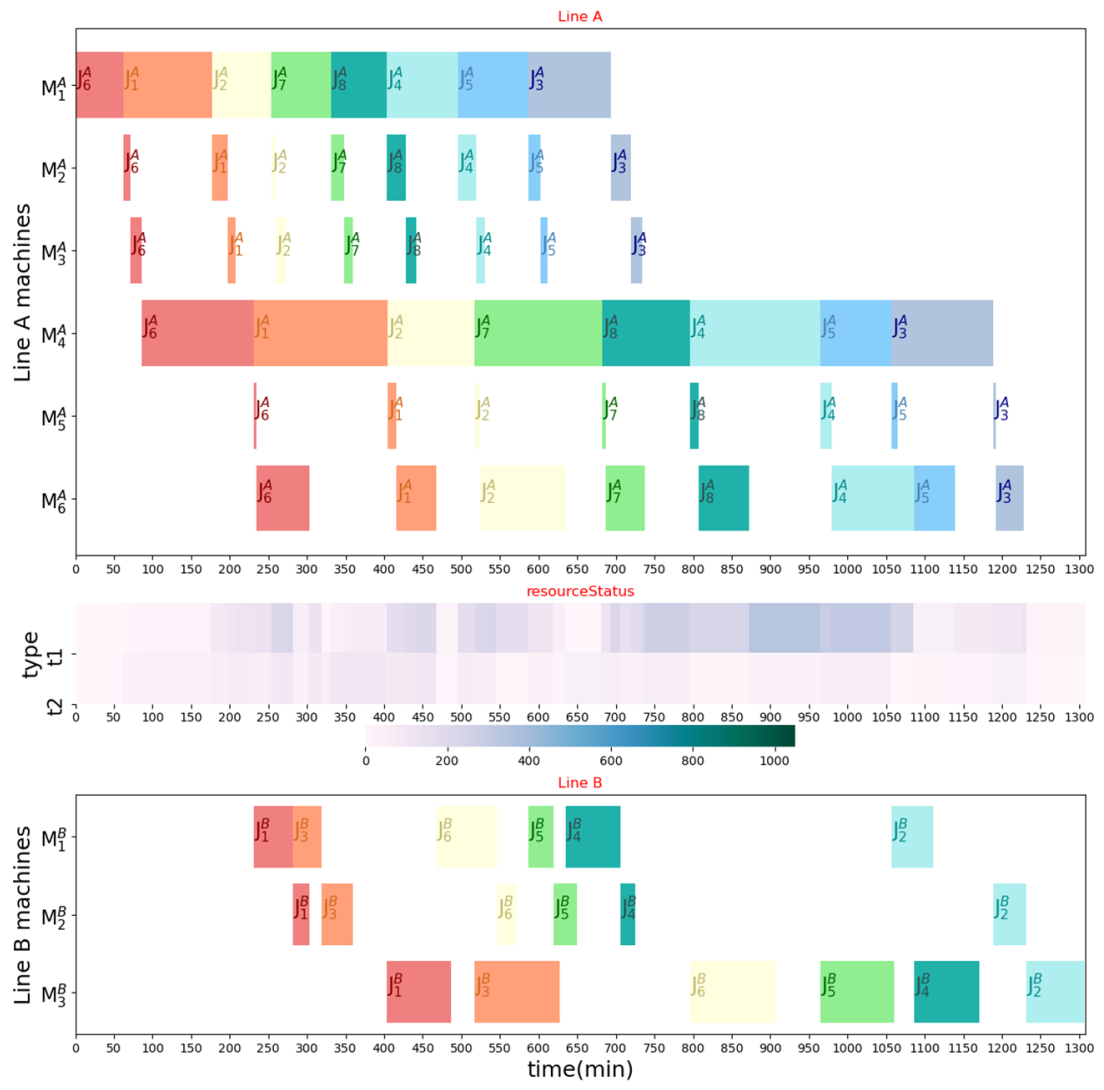

(c)

Figure 5. The Gantt chart of the best schedule of both lines and the availability of the two types of circular materials: (a) FCFS; (b) Campbell-Dudek; (c) GA.

Following the same experimental protocol applied in Section 3.1, the FCFS was conducted one time, the Campbell-Dudek heuristic was iteratively applied 10 times, and the GA was executed for 30 independent runs. The descriptive statistics with these experiments are reported in Table 6. It is seen that the comparative performance was consistent across all of the 10 problem instances. The makespan obtained by GA was the best, followed by Campbell-Dudek heuristic, and the one obtained by FCFS was the longest. For the average makespan and the Std over multiple runs, GA always obtained prevailing results as compared to the Campbell-Dudek heuristic. At the bottom of Table 6, we show the mean statistics over the 10 problem instances. It implies that for the best-case analysis, GA can save 164 and $28 \mathrm{~min}$ in makespan as compared to the FCFS and Campbell-Dudek heuristic, respectively. While for the average case, GA can save 159 and 46 min in makespan as compared to its two competitors. To provide more insight, we transformed the absolute makespan difference to a relative gap ratio, which is defined as the ratio between the absolute makespan difference and the FCFS makespan. We can conclude that the makespan of the Campbell-Dudek heuristic was 9.5\% shorter than that of FCFS in the best case and 7.9\% in the average case, while the makespan of GA was $11.5 \%$ shorter than that of FCFS in the best case and $11.2 \%$ in the average case. For comparison between the Campbell-Dudek heuristic and GA, the makespan of GA was 2.2\% shorter than that of the Campbell-Dudek heuristic in the best case and 3.5\% in the average case. From the reported standard deviation across multiple runs on each of the ten problem instances, we claim that GA is robust against diverse production scenarios of the CRMP problem and delivers consistent-quality results as compared to FCFS and the Campbell-Dudek heuristic. 
Table 6. The experimental result of the three competing methods with the synthetic dataset.

\begin{tabular}{cccccccc}
\hline & FCFS & \multicolumn{3}{c}{ Campbell-Dudek } & \multicolumn{3}{c}{ GA } \\
\hline Instance No. & Best & Best & Average & Std & Best & Average & Std \\
\hline 1 & 1440 & 1271 & 1323.60 & 28.89 & 1254 & 1258.83 & 9.80 \\
2 & 1398 & 1238 & 1257.50 & 17.26 & 1211 & 1211.60 & 2.24 \\
3 & 1434 & 1282 & 1282.60 & 1.20 & 1244 & 1249.07 & 7.00 \\
4 & 1431 & 1291 & 1319.40 & 31.71 & 1231 & 1233.57 & 3.03 \\
5 & 1440 & 1295 & 1321.20 & 20.29 & 1284 & 1285.67 & 5.61 \\
6 & 1417 & 1290 & 1301.40 & 9.37 & 1259 & 1266.67 & 5.14 \\
7 & 1474 & 1347 & 1366.00 & 15.72 & 1308 & 1316.00 & 7.36 \\
8 & 1465 & 1323 & 1348.60 & 25.09 & 1302 & 1305.43 & 5.79 \\
9 & 1364 & 1299 & 1317.00 & 14.93 & 1270 & 1273.53 & 3.96 \\
10 & 1407 & 1281 & 1299.30 & 11.98 & 1272 & 1278.07 & 6.30 \\
\hline Average & 1427.00 & 1291.70 & 1313.66 & 17.64 & 1263.50 & 1267.84 & 5.62 \\
\hline Gap ratio & - & $9.5 \%$ & $7.9 \%$ & & $11.5 \%$ & $11.2 \%$ & \\
\hline
\end{tabular}

\subsection{Sensitivity Analysis}

To analyze the robustness and characteristics of the proposed GA, the sensitivity analysis was conducted as follows. The critical parameters of CRMP that influenced the performance of the algorithm were the number of machines and the number of jobs for each production line. We thus executed the GA with combinations of various parameter setting values. We first fixed the value of the number of machines in the two lines $\left(m_{1}=6\right.$, $\left.m_{2}=3\right)$ and varied the number of jobs with different values $\left(j_{1}=4, j_{2}=3 ; j_{1}=8, j_{2}=6\right.$; $\left.j_{1}=16, j_{2}=12 ; j_{1}=32, j_{2}=24\right)$. Secondly, we fixed the value of the number of jobs in the two lines $\left(j_{1}=8, j_{2}=6\right)$ and varied the number of machines with different values $\left(m_{1}=4\right.$, $m_{2}=2 ; m_{1}=6, m_{2}=3 ; m_{1}=8, m_{2}=4 ; m_{1}=10, m_{2}=5$ ). The statistics over 30 independent runs of GA for each problem instance are listed in Table 7. To realize the performance of GA, the integer programming proposed in Section 2.2 was solved to optimality or the best feasible bound obtained at a maximal CPU time set to $24 \mathrm{~h}(86,400 \mathrm{~s})$. The computed result of the integer programming on the same dataset is shown in Table 8.

Table 7. The sensitivity analysis of GA.

\begin{tabular}{ccccc}
\hline $\boldsymbol{m}_{\mathbf{1}}-\boldsymbol{m}_{\mathbf{2}}-\boldsymbol{j}_{\mathbf{1}} \boldsymbol{j}_{\mathbf{2}}$ & Best & Average & Std & CPU Time \\
\hline $6-3-4-3$ & 687 & 687 & 0 & 0.3742 \\
$6-3-8-6$ & 1307 & 1315 & 8.05 & 1.2764 \\
$6-3-16-12$ & 2370 & 2370.40 & 2.15 & 4.6592 \\
$6-3-32-24$ & 4497 & 4501.27 & 4.84 & 16.9457 \\
\hline $4-2-8-6$ & 1277 & 1277 & 0 & 0.9479 \\
$6-3-8-6$ & 1307 & 1315 & 8.05 & 1.2764 \\
$8-4-8-6$ & 1201 & 1201 & 0 & 1.5196 \\
$10-5-8-6$ & 1408 & 1408 & 0 & 2.0288 \\
\hline
\end{tabular}

It is seen that the number of jobs $\left(j_{1}\right.$ and $\left.j_{2}\right)$ was a more influential parameter than the number of machines $\left(m_{1}\right.$ and $\left.m_{2}\right)$. When the number of jobs increased, the best and average makespan from multiple runs of GA may differ, and the consumed CPU time increased due to a longer length $\left(j_{1}+j_{2}\right)$ needed for encoding the chromosome. Similarly, the increasing complexity makes the CRMP intractable. The exact solution can only be obtained for small problems $\left(j_{1}=4, j_{2}=3 ; j_{1}=8, j_{2}=6\right)$. For larger-sized problems $\left(j_{1}=16, j_{2}=12 ; j_{1}=32\right.$, $\left.j_{2}=24\right)$, no exact solution can be obtained within $86,400 \mathrm{~s}$, and only the best feasible bounds can be returned. For small problems, GA obtained the global optimal solutions as the exact ones. For larger-sized problems, the solutions evolved by GA were significantly better than the best feasible bounds reported by the exact method at the maximal CPU time, validating the robustness of our GA against the number of jobs. 
Table 8. The exact solution or the best feasible bound obtained at the maximal CPU time.

\begin{tabular}{cccc}
\hline $\boldsymbol{m}_{\mathbf{1}}-\boldsymbol{m}_{\mathbf{2}}-\boldsymbol{j}_{\mathbf{1}}-\boldsymbol{j}_{\mathbf{2}}$ & Exact Solution & Feasible Bound & CPU Time \\
\hline $6-3-4-3$ & 687 & & 0.5924 \\
$6-3-8-6$ & 1307 & 2427 & $66,667.15$ \\
$6-3-16-12$ & & 4619 & 86,400 \\
$6-3-32-24$ & & 1277 & 86,400 \\
\hline $4-2-8-6$ & & & 86,400 \\
$6-3-8-6$ & 1307 & & $66,667.15$ \\
$8-4-8-6$ & 1201 & 1408 & $74,804.99$ \\
$10-5-8-6$ & & & 86,400 \\
\hline
\end{tabular}

As shown in the second part of experiments in Tables 7 and 8 , the number of machines was not as critical as the number of jobs. When we varied the number of machines, the best and the average makespan from multiple runs of GA were the same except for the case $\left(m_{1}=6, m_{2}=3 ; j_{1}=8, j_{2}=6\right)$. The consumed CPU times were also very near to each other because the length $\left(j_{1}+j_{2}\right)$ required for encoding the chromosome was the same. For the exact method, the required computation time to obtain the exact solution was very close to the maximal CPU time. When the exact solution cannot be resolved within $86,400 \mathrm{~s}$, the reported best feasible bound is likely the exact solution, which was also the best solution obtained by the GA. Again, this phenomenon validates the robustness of the proposed GA against the variations on the number of machines.

\section{Discussions}

The circular economy and circular manufacturing are re-design processes for materials, resources, operations, reuse, recycling, recovery, and end-of-life disposal to create a closedloop economy such that waste is minimized. This paper studies the re-design of parallel production lines from a rubber manufacturer and how circular economy helps improve the effectiveness and efficiency of the use of circular materials. We propose a genetic algorithm that is intentionally customized to the production re-design. It has been shown that the customized genetic algorithm prevailed in minimizing the makespan as compared to classic FCFS and Campbell-Dudek heuristic, which do not consider and utilize the manufacturing re-design features. These findings are important for both academia and in practice. Hence, this study has the following managerial implications. (1) Circular economy is achievable by re-designing production process, considering the interests and expectations of stakeholders including government agents, international organizations, customers, manufactures, and their partners. (2) Circular economy in rubber manufacturing is an opportunity for creating additional benefits, not just in reducing waste and cost, but also in adding value by producing circular products and enhancing corporate social image. (3) Classic scheduling methods may not be as efficient as for traditional manufacturing problems. New approaches based on appropriate information technologies that fully utilize the re-design context of circular manufacturing are prevailing. (4) To promote the benefits of circular manufacturing to a large extent, including materials, production, recycle, remanufacturing, reuse, logistics, and end-of-life products, cooperation between all stakeholders is necessary to build the circular economy eco-system.

\section{Conclusions}

In this paper we have presented a mathematical formulation for the CRMP problem, which is reflected from the real context of rubber circular manufacturing. The manufacturer has two main production lines. The first line produces main rubber products, while the second line reuses the scrap rubber granulates and strips produced by the first line and mixes them with other materials to manufacture light-weight rubber works. A GA customized to the CRMP context is developed. The experimental results with both real and synthetic datasets demonstrate that the GA is applicable to the real CRMP context and is robust enough to tackle comprehensive synthetic datasets. Our GA significantly surpasses 
the FCFS and the Campbell-Dudek heuristic by delivering the minimum makespan of CRMP datasets. Our paper is the first attempt in the literature to address the two-line flowshop scheduling under multiple resource constraints. The empirical experimental results show that the proposed model and the GA method can facilitate the rubber circular manufacturing practice and achieve efficient production to save both production time and material cost.

The results of the current study are limited to a single case study of rubber manufacturer. The data collected from the real site may not reflect the production context of other rubber manufacturers. Our future research study will focus on the simulations of uncertain production parameters such that the achieved solutions will generalize to a broad range of circular manufacturing. Moreover, our current approach deals with the reuse of waste in intermediate processes. The process of reverse logistics, which allows the reuse, recycling, and final disposal of product to go backward in the chain for possible re-manufacture, is worth further studying. The adoption of appropriate information technologies to facilitate circular economy considering the interests and expectations of all stakeholders is also a promising research direction.

Author Contributions: Conceptualization, P.-Y.Y., H.-M.C., Y.-L.C. and Y.-C.W.; methodology, P.-Y.Y. and H.-M.C.; software, P.-Y.Y., Y.-L.H. and R.-F.D.; validation, P.-Y.Y., Y.-L.H. and R.-F.D.; writingoriginal draft preparation, P.-Y.Y. and H.-M.C.; writing—review and editing, P.-Y.Y.; visualization, P.-Y.Y. and Y.-L.H.; funding acquisition, P.-Y.Y. and R.-F.D. All authors have read and agreed to the published version of the manuscript.

Funding: This research was funded by Ministry of Science and Technology of ROC, grant number MOST 107-2410-H-260-015-MY3 and MOST 107-2420-H-260-002-HS3.

Institutional Review Board Statement: Not applicable.

Informed Consent Statement: Not applicable.

Data Availability Statement: Restrictions apply to the availability of these data. Data was obtained from Sun Rubbers Co. and are available from the authors with the permission of Sun Rubbers Co.

Conflicts of Interest: The authors declare no conflict of interest. The funders had no role in the design of the study; in the collection, analyses, or interpretation of data; in the writing of the manuscript, or in the decision to publish the results.

\section{References}

1. Su, B.; Heshmati, A.; Geng, Y.; Yu, X. A review of the circular economy in China: Moving from rhetoric to implementation. J. Clean. Prod. 2013, 42, 215-227. [CrossRef]

2. Geissdoerfer, M.; Savaget, P.; Bocken, N.; Hultink, E. The circular economy-A new sustainability paradigm. J. Clean. Prod. 2017, 143, 757-768. [CrossRef]

3. Lieder, M.; Rashid, A. Towards circular economy implementation: A comprehensive review in context of manufacturing industry. J. Clean. Prod. 2016, 115, 36-51. [CrossRef]

4. Kumar, V.; Sezersan, I.; Garza-Reyes, J.A.; Gonzalez, E.D.R.S.; Al-Shboul, M.A. Circular economy in the manufacturing sector: Benefits, opportunities and barriers. Manag. Decis. 2019, 57, 1067-1086. [CrossRef]

5. González-Sánchez, R.; Settembre-Blundo, D.; Ferrari, A.M.; García-Muiña, F.E. Main dimensions in the building of the circular supply chain: A literature review. Sustainability 2020, 12, 2459. [CrossRef]

6. Fontana, A.; Barni, A.; Leone, D.; Spirito, M.; Tringale, A.; Ferraris, M.; Reis, J.; Goncalves, G. Circular economy strategies for equipment lifetime extension: A systematic review. Sustainability 2021, 13, 1117. [CrossRef]

7. Usama Awan, U.; Sroufe, R.; Shahbaz, M. Industry 4.0 and the circular economy: A literature review and recommendations for future research. Bus. Strategy Environ. 2021. [CrossRef]

8. Alhawari, O.; Awan, U.; Bhutta, M.K.S.; Ulku, M.A. Insights from Circular Economy Literature: A Review of Extant Definitions and Unravelling Paths to Future Research. Sustainability 2021, 13, 859. [CrossRef]

9. UNEP. Towards a Green Economy: Pathways to Sustainable Development and Poverty Eradication. 2011. Available online: https:/ / www.unep.org/greeneconomy/ (accessed on 20 January 2021).

10. 2011 Yearbook of GTPO. Available online: https:/ / www.greentrade.org.tw/publication/185/ (accessed on 20 January 2021).

11. Rubber Journal Asia (RJA) News. Recycling: Tyre Sector Tackles Circularity of Rubber. Available online: http:/ / rubberjournalasia. com/recycling-tyre-sector-tackles-circularity-of-rubber/ (accessed on 20 January 2021). 
12. Wielgosiński, G.; Namiecińska, O.; Czerwińska, J. Environmental impact of emissions from incineration plants in comparison to typical heating systems. E3S Web Conf. 2018, 28, 01038. [CrossRef]

13. Sathiskumara, C.; Karthikeyan, S. Recycling of waste tires and its energy storage application of by-products-A review. Sustain. Mater. Technol. 2019, 22, e00125. [CrossRef]

14. Evonik Industries News. Efficient Recycling of Scrap Rubber-From Scrap Tires to Cow Mattresses. Available online: https: / / www.vestenamer.com/en/additive-rubber-recycling/from-scrap-tires-to-cow-mattresses/ (accessed on 20 January 2021).

15. 2020 National Statistics Report, Directorate-General of Budget, Accounting and Statistics, Taiwan. Available online: https: / / eng.dgbas.gov.tw/ (accessed on 20 January 2021).

16. Garey, M.R.; Johnson, D.S. Computers and Intractability: A Guide to the Theory of NP-Completeness. A Series of Books in the Mathematical Sciences; Freeman, W.H., Ed.; W. H. Freeman \& Co.: New York, NY, USA, 1979; ISBN 0-7167-1045-5.

17. Johnson, S.M. Optimal two and three stage production schedules with set-up times included. Naval Res. Logist. Q. 1954, 1, 61-68. [CrossRef]

18. Campbell, H.G.; Dudek, R.A. A heuristic algorithm for the $\mathrm{n}$ job, $\mathrm{m}$ machine sequencing problem. Manag. Sci. 1970, 16, B630-B637. [CrossRef]

19. Nawaz, M.; Enscore, E.; Ham, I. A heuristic algorithm for the m-machine, n-job flow-shop sequencing problem. Int. J. Manag. Sci. 1982, 11, 91-95. [CrossRef]

20. Laribi, I.; Yalaoui, F.; Belkaid, F.; Sari, Z. Heuristics for solving flow shop scheduling problem under resources constraints. IFAC-PapersOnLine 2016, 49, 1478-1483. [CrossRef]

21. Osman, I.H.; Potts, C.N. Simulated annealing for permutation flow-shop scheduling. Omega 1989, 17, 551-557. [CrossRef]

22. Murata, T.; Ishibuchi, H.; Tanaka, H. Genetic algorithms for flowshop scheduling problems. Comput. Ind. Eng. 1996, 30, 1061-1071. [CrossRef]

23. Reeves, C.R. A genetic algorithm for flowshop sequencing. Comput. Oper. Res. 1995, 22, 5-13. [CrossRef]

24. Eksioglu, B.; Eksioglu, S.D. A tabu search algorithm for the flowshop scheduling problem with changing neighborhoods. Comput. Ind. Eng. 2008, 54, 1-11. [CrossRef]

25. Goldberg, D.E. Genetic Algorithms: Search, Optimization and Machine Learning; Addison-Wesley: Reading, MA, USA, 1989.

26. Deb, K. Introduction to genetic algorithms for engineering optimization. In New Optimization Techniques in Engineering. Studies in Fuzziness and Soft Computing; Onwubolu, G.C., Babu, B.V., Eds.; Springer: Berlin/Heidelberg, Germany, 2004; Volume 141, pp. 13-51.

27. Lee, C.K.H. A review of applications of genetic algorithms in operations management. Eng. Appl. Artif. Intell. 2018, 76, 1-12. [CrossRef]

28. Holland, J. Adaptation in Natural and Artificial Systems, Reprint ed.; Originally Published in 1975; The MIT Press: Cambridge, $\mathrm{UK}, 1992$. 